\title{
Standardized Baseline Human Corneal Subbasal Nerve Density for Clinical Investigations With Laser-Scanning in Vivo Confocal Microscopy
}

\begin{abstract}
Marlen Parissi, Georgios Karanis, Stefan Randjelovic, Johan Germundsson, Enea Poletti, Alfredo Ruggeri, Tor Paaske Utheim and Neil Lagali
\end{abstract}

\section{Linköping University Post Print}

\section{Tweet}

N.B.: When citing this work, cite the original article.

Original Publication:

Marlen Parissi, Georgios Karanis, Stefan Randjelovic, Johan Germundsson, Enea Poletti, Alfredo Ruggeri, Tor Paaske Utheim and Neil Lagali, Standardized Baseline Human Corneal Subbasal Nerve Density for Clinical Investigations With Laser-Scanning in Vivo Confocal Microscopy, 2013, Investigative Ophthalmology and Visual Science, (54), 10, 7091-7102. http://dx.doi.org/10.1167/iovs.13-12999

Copyright: Association for Research in Vision and Ophthalmology (ARVO) http://www.arvo.org/

Postprint available at: Linköping University Electronic Press http://urn.kb.se/resolve?urn=urn:nbn:se:liu:diva-102405 


\section{Standardized Baseline Human Corneal Subbasal Nerve Density for Clinical}

Investigations with Laser-scanning in Vivo Confocal Microscopy

Marlen Parissi ${ }^{1,2}$, Georgios Karanis ${ }^{3}$, Stefan Randjelovic ${ }^{2}$, Johan Germundsson ${ }^{3}$, Enea Poletti ${ }^{4}$, Alfredo Ruggeri ${ }^{4}$, Tor Paaske Utheim ${ }^{1,2,5}$, and Neil Lagali ${ }^{3}$

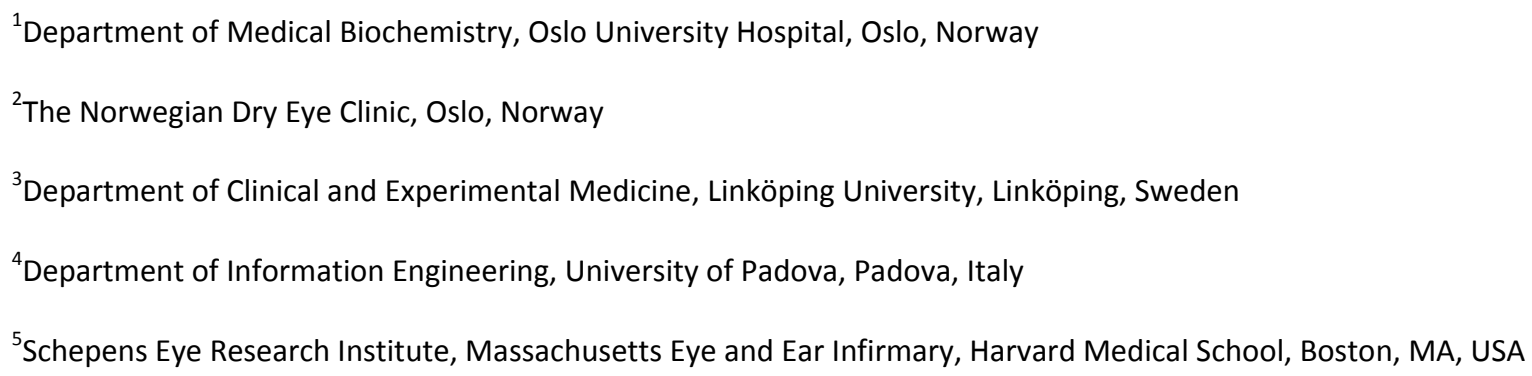

Abstract word count: 245

None of the authors have any proprietary/financial interest to disclose.

Running head: Baseline corneal subbasal nerve density

Sources of Funding: Funding from the Cronqvist Foundation, the Swedish Research Council, and Princess Margaretas Foundation for the Visually Impaired to NL, and funding from the Norwegian Research Council to MP.

None of the authors have any proprietary/financial interest to disclose.

Corresponding author:

Neil Lagali, PhD

Department of Clinical and Experimental Medicine - Ophthalmology

Faculty of Health Sciences

Linköping University

58183 Linköping, Sweden

neil.lagali@liu.se

Tel +46101034658

Fax +46101033065 


\section{ABSTRACT}

Purpose: To establish a baseline value for central corneal subbasal nerve density in a large, healthy cohort.

Methods: 106 healthy volunteers (207 eyes) underwent full ophthalmic examination including laser-scanning in vivo confocal microscopy (IVCM) of the central cornea. Images of the corneal subbasal nerve plexus were acquired and analyzed based on defined criteria. Nerve tracing was performed by two human observers and by a fully automated method. Subbasal nerve density was stratified by eye, observer, tracing method, calculation method, and age group. Association of nerve density with age was examined by linear regression and population distribution was examined by nonlinear regression.

Results: 892 distinct, high quality images of the subbasal nerve plexus (mean 4.3 images/eye) were analyzed from 207 eyes. An overall mean central subbasal nerve density of $19 \mathrm{~mm} / \mathrm{mm}^{2}$ was found in 106 subjects aged $15-88$ years, independent of eye, gender, or nerve tracing method, while the standard deviation was a consistent $4-5 \mathrm{~mm} / \mathrm{mm}^{2}$. Subbasal nerve density followed a normal, Gaussian distribution, and correlated negatively with age, with a mean decline of $0.25 \%-0.30 \%$ per year, independent of eye, observer, or nerve tracing method. Moreover, the use of automated tracing techniques and randomized sampling may improve the speed and reproducibility of subbasal nerve density assessment for clinical applications.

Conclusions: A baseline human corneal subbasal nerve density has been determined by laser-scanning IVCM using rigorous methods. The methods and results could aid in the future assessment of corneal nerves in various patient populations. 


\section{Introduction}

The use of in vivo confocal microscopy (IVCM) for rapid, non-invasive clinical assessment of the cornea has grown substantially in recent years. Among the increasing number of clinical studies using IVCM are frequent reports describing corneal nerves in health and pathology (for a review of the subject see References 1,2). These studies overwhelmingly focus on corneal nerves which are confined to a thin plexus located at the junction of the basal epthelium and the anterior aspect of Bowman's layer. ${ }^{3}$ The nerve fiber bundles comprsing this plexus (hereafter termed 'subbasal nerves') are considered an important anatomical landmark, not only because of their confinement to a specific layer (which renders them amenable to visualization by confocal optical sectioning), but also because they give rise to epithelial innervation and the intraepithelial nerve endings terminating at the ocular surface. ${ }^{3,4}$ Therefore, by extension, the subbasal nerves can directly influence (or be influenced by) the corneal epithelium, which in turn is a vital part of the ocular immune system. While the importance of epithelial innervation on the health and functioning of the epithelium, tear film, and cornea is firmly established, ${ }^{5}$ a growing body of research is also revealing that the subbasal nerves can serve as a proxy for assessing the health or pathology of the cornea ${ }^{1,2}$ and even gauging the severity of non-ocular conditions, such as peripheral neuropathy in diabetes mellitus ${ }^{6}$ or rheumatoid arthritis. $^{7}$

As new relationships between corneal subbasal nerves and various pathologic entities (such as diabetes, ${ }^{6,8}$ limbal stem cell deficiency, ${ }^{9,10}$ aniridia, ${ }^{11}$ dry eye disease ${ }^{12,13}$ herpetic keratitis, ${ }^{14}$ Fuchs' dystrophy, ${ }^{15,16}$ etc.) are being discovered, more sophisticated techniques for subbasal nerve image acquisition, ${ }^{17-19}$ visualization, ${ }^{20,21}$ and quantification ${ }^{22}$ are also being reported. Translating these discoveries from populationbased studies into useable clinical information for assessment, prognosis and diagnosis of a condition on an individual patient basis, however, has received comparatively little attention. In order to be of value for clinical decision-making, indicators based on subbasal nerves should be quantitative, sensitive, and specific. Unfortunately at present, there is a lack of consensus regarding the even most basic question regarding subbasal nerves, namely their density in a normal, healthy central cornea. This question is of fundamental importance as it establishes a baseline against which a 'pathological' level can be defined. Part of the problem is the use of both slit scanning and laser scanning confocal systems for imaging, which can yield widely disparate results ${ }^{1,6}$, with the laser system providing better axial resolution and higher contrast, generally rendering more subbasal nerves visible. ${ }^{23,24}$ Even among studies using laser-scanning IVCM, however, a lack of standardization of methods for subject selection (in terms of age distribution), image acquisition (volume or sequence scan mode), image selection (number of images to analyze, ${ }^{25}$ dense versus representative regions), and image analysis (number and experience of observers, nerve definition criteria, masking and randomization), as well as inadequate methodological reporting, have resulted in wide discrepancies and bias-prone estimates for even this most basic parameter.

Nevertheless, subbasal nerve density (defined as total length of all subbasal nerves visible in vivo per unit area of the cornea, expressed in $\mu \mathrm{m} / \mathrm{mm}^{2}$ or $\mathrm{mm} / \mathrm{mm}^{2}$ ) can still be considered to be the most 'mature' parameter for quantitative analysis of the subbasal nerve plexus. Other interesting and potentially clinically applicable parameters, such as subbasal nerve branch density and tortuosity, are less frequently reported ${ }^{1,6,26,27}$ and even less standardized than subbasal nerve density.

A logical first step in addressing these issues would be to first focus on a single parameter such as subbasal nerve density, standardize the procedures for its determination, and report its value (and statistical distribution of this value) in a normal, healthy population. Despite discrepancies in the literature, some 
generally accepted guidelines for image selection and analysis have implicitly emerged that can be adopted and formally stated as explicit criteria. The current study was therefore undertaken to develop a standardized approach to quantification of subbasal nerve density, and apply this to a normal, healthy population of subjects. We hypothesized that a rigorous approach to study design, image acquisition, selection, analysis, and reporting would result in subbasal nerve density values that differed from previously reported values in healthy cohorts.

This study aims to answer a several questions of importance for confocal microscopy of corneal nerves in research and clinical practice:

1. What is the central corneal subbasal nerve density (and its statistical distribution) in the human cornea measured in vivo, in a large cohort?

2. How does subbasal nerve density vary with age, gender, and eye?

3. What is the best criterion for image selection and how many images should be selected from a given cornea to determine a single subbasal nerve density value?

\section{Materials and methods}

\section{Subjects}

With ethical approval from the regional ethical review board in Linköping, Sweden, 115 healthy volunteer subjects were recruited for this study. Recruited subjects were persons accompanying patients at the Ophthalmology Department, Linköping University. Prior to examination, each subject provided signed informed consent to participate in the study, and the study was conducted according to the tenets of the Declaration of Helsinki. Subjects with diabetes, with a history of contact lens wear, those using ocular medication or those with prior corneal surgery, symptoms consistent with ocular irritation, discomfort, or dry eye were excluded. Additionally, subjects with unilateral corneal pathology or any history of corneal pathology or symptomatology were excluded. Subjects additionally underwent a full ophthalmic examination to rule out corneal or other potentially confounding ocular pathology. Examination was bilateral and included slit lamp biomicroscopy, intraocular pressure, visual acuity, in vivo confocal microscopy and anterior segment optical coherence tomography. In the later phases of recruitment, individuals were approached based on a target recruitment of approximately 25 individuals from each of four age groups (15-30y, 31-45y, 46-60y, 61+y) and a requirement for sufficient representation of the eldest age group. $^{28}$

In vivo confocal microscopy

A laser scanning in vivo confocal microscope (IVCM; Heidelberg Retinal Tomograph 3 with Rostock Corneal Module, Heidelberg Engineering, Heidelberg, Germany) was used to examine all study subjects. The microscope was outfitted with a 63x/0.95 NA immersion objective lens (Zeiss, Oberkochen, Germany), to provide images representing an en face view of a $400 \mu \mathrm{m} \times 400 \mu \mathrm{m}$ corneal area. Following manufacturer guidelines, a drop of tear gel (Viscotears, carbomer 0.2\%, Dr Mann Pharma, Berlin, Germany), was applied to the objective lens, covered with a disposable sterile cap (TomoCap ${ }^{\circledR}$, polymethylmethacrylate) and a second drop of tear gel was placed on the cap. Using manual lateral and axial controls, the gel-coated cap was 
brought into contact with the central cornea of a topically anesthetized cornea (Tetracaine hydrochloride $1 \%$, Chauvin Pharmaceuticals Ltd., UK).

Axial depth was adjusted using a motorized joystick control, and was first set to image an epithelial wing cell layer. When this layer appeared in the real-time display window, scanning was initiated in either 'sequence scan' or 'volume scan' mode. Sequence and volume scans were used because it was not known a priori which scan mode would produce the greatest number of distinct, high-quality images of subbasal nerves. The volume scan mode yields a finer axial spacing between images, but is limited to a single field of view during scanning and therefore takes considerably longer to scan a large area of the subbasal plexus than the sequence scan mode. In the sequence scan mode, the axial depth was first manually adjusted to visualize the subbasal nerve plexus, then image acquisition of 100 consecutive image frames ( 8 frames $/ \mathrm{sec}$ ) was initiated. During acquisition, sequence mode allows the lateral positioning controls to be manually adjusted to sweep the field of view over a larger area of the central corneal subbasal nerve plexus. This manual sweeping was done in sequence mode, to obtain a wider sampling of the central corneal subbasal nerve plexus. In the volume scan mode, image acquisition was initiated with epithelial wing cells initially in view, and the acquisition stopped automatically when the anterior stroma was in view (40 images were automatically acquired over an axial depth of $80 \mu \mathrm{m}$ ). In the case of both volume and sequence scans, several repeated scans were performed to image subbasal nerves in different regions of the central cornea. During examination, care was taken to minimize pressure of the Tomocap ${ }^{\circledR}$ on the cornea by manual axial adjustment of the entire corneal module, to avoid pressure-induced striation artifacts in images. ${ }^{21}$ In all cases, the automatic brightness adjustment setting on the HRT3-RCM system was used.

Image selection

Three experienced observers developed the criteria for image selection. Patients were first assigned a random numeric code based on alphabetical sorting of the group by patient surname. Images from each eye were then viewed, with observers masked to the patient data such as age and gender. Images were selected from sequence or volume scans based on their quality according to the following criteria:

1. absence of motion artifact

2. absence of pressure-induced artifact

3. providing an en face, non-oblique view

4. high-contrast images with clear delineation of nerves from the background

5. location - central cornea, distinct nerves without overlap from other selected images, and excluding the central whorl region where a clear spiral pattern and/or characteristic 'white dots' ${ }^{17,29}$ are visible in a single frame

6. Where images of the same nerve pattern at different depths existed, the image with the greatest contrast/visibility of nerves was chosen.

No specific criteria were set for the number of distinct images to select; instead, all images satisfying these criteria were chosen. Practically, however, the above requirement for distinct nerves without visible overlap (or very minor overlap at image borders, less than 10\%) and the limited total examination time per eye (about 5 min/eye) limited the number of images extracted from a given eye. Each extracted image was coded with a unique identifier (indicating patient number, eye, scan number, and image number) saved in TIFF format. Image selection was performed by two trained observers who did not perform the confocal examination. 
Manual nerve tracing

Guidelines for nerve tracing were developed by three experienced observers. Coded images were traced by two independent observers during several sessions over a two-week period, at an off-site location. Tracing was performed by two trained observers who did not perform the confocal examination. Manual nerve tracing was performed on raw TIFF images using ImageJ software (version 1.45s, Rasband, W.S., ImageJ, U. S. National Institutes of Health, Bethesda, Maryland, USA, http://imagej.nih.gov/ij/, 1997-2012) with the NeuronJ plugin, ${ }^{30}$ as we have described previously. ${ }^{31}$ Images used for tracing were raw images that were not brightness or contrast adjusted or post-processed in any way. Images were analyzed by both observers on the same computer screen without changing screen settings. The display size of the image was first increased to $150 \%$ in NeuronJ, and all nerves and branches in an image were traced. Where contrast between a nerve segment and the background reduced the ability to determine whether a nerve should be traced (faint nerves or interconnecting branches), the following criterion was used: a nerve segment was included in the tracing if along its length at least $75 \%$ of the nerve was visible. In other words, thin, lowcontrast nerve segments where more than $25 \%$ of the nerve segment was not visible were excluded. Dendritic cells, their long dendrites, and dendritic-like features were also excluded from nerve tracings. The total length of all tracings in an image was determined in NeuronJ, and converted to a value in $\mathrm{mm}$. This value, divided by the frame area of $0.16 \mathrm{~mm}^{2}(400 \times 400 \mu \mathrm{m})$ yielded nerve density in $\mathrm{mm} / \mathrm{mm}^{2}$. Nerve densities were recorded in a spreadsheet. After all nerve densities were recorded, the codes were unmasked and data was arranged according to eye, subject number, gender, and age.

\section{Automatic nerve tracing}

Additionally, nerves were independently automatically traced with a modified version of an algorithm designed for the automatic recognition of corneal nerve structures. ${ }^{22}$ The acquired images were first normalized and enhanced in luminosity and contrast. The nerves were then recognized by applying a tracing algorithm that links sparse seed points into continuous structures via a graph-search technique. The technique includes multi-scale matched template filtering to enhance nerve visibility and post-processing procedures to remove false recognitions. The procedure requires no user intervention and the average run time per image is 2.5 minutes.

In order to obtain a measure of nerve length comparable to the one obtained with NeuronJ, the final nerve tracing was processed in the same way as in NeuronJ, i.e., using not only the image values at the vertex coordinates, but also image values at points between vertices. The number of additional points included in each between-vertices interval was equal to 5, the NeuronJ default. For each pair of vertices, the additional points were sampled along a straight line connecting the vertices and bilinear interpolation was used to compute the image values at these points.

\section{Quantitative and statistical analysis}

Inter-observer and inter-method (manual versus automatic) differences in nerve density were assessed using all subbasal nerve images (several images per eye). The Bland-Altman method ${ }^{32}$ was used to detect observer bias and to determine the $95 \%$ limits of agreement (LOA). For comparison of subbasal nerve density across different age groups, only one density value per eye was used, and the analysis was stratified by eye. As several images (and hence densities) were available for a given eye, different methods were used to compute the single nerve density value to be used for each eye in the analysis. These methods were: 
average (mean of density values from all traced images of a given eye), maximum (maximum nerve density image), minimum (minimum nerve density image), 1 random (density from one randomly selected image from the eye), 2 random (mean density of two randomly selected images from the eye), 3 random (mean density of three randomly selected images from the eye), and 4 random (mean density of four randomly selected images from the eye).

Association of subbasal nerve density with age and inter-eye correlations were tested with the Pearson Product Moment Correlation test, while differences in nerve density between male and female subjects were tested with independent t-tests. In all cases, results were considered significant at a two-tailed level of $\alpha<0.05$, and normality was tested with the Kolmogorov-Smirnov test. All statistics were performed with commercial statistical software (SigmaStat 3.5 for Windows, Systat Software Inc., Chicago IL, USA). Linear regression analysis was performed using the built-in regression function in Excel (Microscoft Office 2010, Microsoft, Redmond WA, USA), while both linear regression (for age association) and nonlinear regression (for density distribution) were performed in SigmaPlot (version 10 for Windows, Systat Software Inc., Chicago IL, USA). The nonlinear regression model employed a three-parameter Gaussian function (peak height, mean, and standard deviation).

\section{Results}

Subjects

In total, 106 subjects participated in this study, out of a total of 115 who gave signed informed consent. During full ophthalmic examination, 9 patients were excluded due to diffuse corneal haze or discrete scars. 212 eyes of the 106 included subjects were examined on one occasion per subject. IVCM examination data did not yield sufficiently high quality images of subbasal nerves in 5 eyes, resulting in 207 eyes with images suitable for analysis. This final group consisted of 59 female (56\%) and 47 male (44 \%) subjects. Subject demographic characteristics are given in Table 1 . The number of subjects in each age category was roughly equal, with exception of a desired overrepresentation for the eldest age group, in which 37 of the 106 (35\%) of subjects were over 61 years of age.

Inter-observer comparisons

\section{Manual nerve tracing}

From the 207 eyes examined, a total of 892 images of central subbasal nerves were selected for analysis (mean of 4.3 images/eye, range $1-7$ images/eye). One observer completed manual tracing of all 892 images, while the second observer traced one image per eye (randomly chosen), 207 images in total (23.2\%). Analysis of the 207 images traced by both observers with the Bland-Altman method revealed a mean nerve density difference of $0.085 \mathrm{~mm} / \mathrm{mm}^{2}$ between observers (mean absolute difference $0.84 \mathrm{~mm} / \mathrm{mm}$ ), and a $95 \%$ LOA of $\pm 2.44 \mathrm{~mm} / \mathrm{mm}^{2}$ (Fig 1 ). In general, agreement between observers was good, with a linear association of nerve density values with slope of 0.96 (Fig. 2). 
Automatic nerve tracing was performed for all 892 images. From the resulting nerve density values, mean nerve density for each of the 207 eyes was calculated and compared to the mean values from the first observer. Bland-Altman analysis revealed a mean density difference of $0.071 \mathrm{~mm} / \mathrm{mm}^{2}$ between manual and automatic methods (mean absolute difference $1.77 \mathrm{~mm} / \mathrm{mm}^{2}$ ), and a $95 \%$ LOA of $\pm 4.52 \mathrm{~mm} / \mathrm{mm}^{2}$ (Fig 1 ). In general, agreement between manual and automatic nerve density determination was good, with a linear association of nerve density values with slope of 0.91 (Fig. 2).

In absolute terms, the mean nerve density from two observers (207 images, one random image/eye) differed from the corresponding automated value by $9.2 \%$ on average (maximum difference $40.5 \%$ ). Taking into account all 892 images analyzed by a single observer, nerve density differed from the automated value by $8.9 \%$ (maximum difference $55.2 \%$ ). Among both observers, nerve density difference was $2.3 \%$ on average (maximum difference 19.5\%).

From Figure 1, three cases with large nerve density differences between human observers were examined visually, with results shown in Figure 3 . When comparing different human observers, tracing errors could be attributed to thin, reduced-contrast nerves traced by only one observer, differences in visualizing/assessing faint nerves where background reflectivity was increased, and erroneous tracing of dendritic cells as nerves. In the case of automated analysis, three cases that corresponded to a relatively large discrepancy between automated and manual results (but with good inter-observer agreement) were analyzed (Figure 4). In these cases, thin, reduced-contrast nerve segments were excluded by the automated method but included by human observers, while dendritic cell dendrites were included by the automated method and excluded by human observers.

Nerve density quantification by age group

For images traced by the first observer, nerve density was calculated by different methods: average, maximum, minimum, and the mean of 1, 2, 3, or 4 random images (see Methods). Additionally, nerve density from the second observer ( 1 random image) was included. Mean and standard deviation of nerve density for the four age groups is shown in Table 1. The method of the taking the average density for all images in a given eye was considered the 'gold standard'. For the other methods of density calculation, the percentage error from the gold standard was determined (Table 2).

The mean nerve density in the eldest age group (61+ years) was decreased relative to the younger groups, independent of eye, calculation method, or observer. Standard deviation of nerve density remained relatively constant (range: $3.5-5.9 \mathrm{~mm} / \mathrm{mm}^{2}$ ), independent of eye, age group, calculation method, or observer.

Error analysis revealed a maximum $+21 \%$ error in density (overestimation relative to the gold standard) by using a single image with the maximum nerve density and a maximum $-20 \%$ error in density (underestimation) by using a single image with the minimum nerve density. Using one randomly selected image per eye, however, resulted in a maximum error of $4.7 \%$ across age groups, eyes, and observers. Using the average density in 2,3 , or 4 random images per eye resulted in maximum errors of $3.1 \%, 1.4 \%$, and $1.2 \%$ across age groups, eyes, and observers, respectively. 
The above density and error analysis was repeated for images processed by the automated method (Table 3). By the automated method, mean nerve density was reduced in the eldest group, independent of eye or calculation method. Range of standard deviation of nerve density was $3.5-6.2 \mathrm{~mm} / \mathrm{mm}^{2}$. A maximum absolute error of $21-23 \%$ in density occurred due to over/underestimation using single images with $\mathrm{max} / \mathrm{min}$ nerve density. Using the density value from a single random image per eye gave a maximum error of $4.7 \%$ in nerve density across age groups and eyes. Using 2, 3, or 4 random images per eye resulted in maximum errors of $4.6 \%, 2.0 \%$, and $1.2 \%$ across age groups and eyes, respectively.

Subbasal nerve density correlation with age

The relationship of nerve density with age was analyzed for the various density calculation methods. This variation is depicted graphically in Figure 5 separately for right and left eyes, using the gold standard method of average nerve density across a mean of 4.3 images/eye. The corresponding plots for the automated analysis are given in Figure 6 . A significant negative correlation was found between average subbasal nerve density and age for right (Pearson $r=-0.198, P=0.04)$ and left $(r=-0.224, P=0.02)$ eyes, based on the manual data. For the automated analysis, the corresponding correlations were $r=-0.244, P=0.01$ (right eyes) and $r=-0.250, P=0.01$ (left eyes).

\section{Linear regression Analysis}

For the different calculation methods, regression analysis was performed to yield the slope (\% change in nerve density per year), $y$-intercept (predicted nerve density at birth), and $R^{2}$ value (Table 4$)$. Using all images obtained (several per eye) or the gold standard average value per eye gave similar values for slope (negative, with a loss of $0.25 \%$ per year) and y-intercept (21-22 $\mathrm{mm} / \mathrm{mm}^{2}$ ), independent of eye. Using the average density in 1 up to 4 random images per eye or using values from the second observer did not substantially affect slope or $y$-intercept for either eye. Using the image with maximum or minimum nerve density, however, affected slope and $y$-intercept to a greater degree. In all cases, $R^{2}$ was relatively low (range $0.03-0.07$ ), reflecting the spread of the density values around the regression line (Figures 5,6 ).

For the automated analysis, slope of the regression line indicated slightly greater loss of nerve density $(0.27$ $-0.31 \%$ per year), while $y$-intercept was consistent with the manual analysis. Results from using a single randomly selected image per eye did not substantially alter the slope or $y$-intercept, relative to the gold standard average value (Table 4).

\section{Distribution of subbasal nerve density}

The overall subbasal nerve density of the entire subject group based on the average density value from 4.3 images/eye was $18.8 \pm 4.8 \mathrm{~mm} / \mathrm{mm}^{2}$ (right eyes) and $19.2 \pm 4.2 \mathrm{~mm} / \mathrm{mm}^{2}$ (left eyes) for manual analysis, and $18.6 \pm 4.8 \mathrm{~mm} / \mathrm{mm}^{2}$, (right eyes) and $19.1 \pm 4.1 \mathrm{~mm} / \mathrm{mm}^{2}$ (left eyes) for automated analysis. The distribution of subbasal nerve density is given in Figure 7 for manual and automated analysis. In all cases a normal distribution was evident, following a standard Gaussian curve with a good fit $\left(R^{2}\right.$ range: $\left.0.82-0.91\right)$ and consistent mean (peak) value (range: $19.0-19.2 \mathrm{~mm} / \mathrm{mm}^{2}$ ). 
Inter-eye correlation and gender association

Subbasal nerve density by the gold standard method in left and right eyes of the same subject was significantly correlated, both by manual $(r=0.229, P=0.02$, Observer $1 ; r=0.222, P=0.03$, Observer 2$)$ and automated $(r=0.280, P=0.005)$ techniques. The mean and SD of inter-eye difference within a subject was -

$0.6 \pm 6.6 \mathrm{~mm} / \mathrm{mm}^{2}$ for manual analysis (single observer, gold standard method) and $0.7 \pm 7.0 \mathrm{~mm} / \mathrm{mm}^{2}$ by the automated method. For both methods, inter-eye subbasal nerve density difference followed a normal distribution, and in both cases 7 subjects (6.7\%) had an inter-eye difference greater than two standard deviations from the mean difference. No association was observed between subject gender and mean subbasal nerve density ( $\mathrm{P}>0.05$ ), independent of observer, eye, or tracing method (manual or automated).

\section{Discussion}

This study represents the largest study to date to document the central corneal subbasal nerve density in a normal, healthy population by laser-scanning IVCM. We endeavored to apply a rigorous approach to acquire, select, and analyze images, and have additionally used multiple observers, tracing methods, and calculation methods to gauge the sensitivity of subbasal nerve density and its age dependence to these various factors. If a single mean value for the 'normal' adult human subbasal nerve density in vivo is to be stated, independent of age, gender, eye, or method of nerve tracing, this value would be $19 \mathrm{~mm} / \mathrm{mm}^{2}$, with a standard deviation of $4-5 \mathrm{~mm} / \mathrm{mm}^{2}$. This standard deviation is surprisingly consistent with age, observer, and analysis method, while the mean value varies with age, from about $20 \mathrm{~mm} / \mathrm{mm}^{2}$ in the youngest adults to $17 \mathrm{~mm} / \mathrm{mm}^{2}$ in the elderly.

As expected, subbasal nerve density had a high inter-eye correlation, and no significant association with gender. In the single prior laser-scanning IVCM study to examine the age and gender correlation with nerve density, ${ }^{28}$ the reported decline of subbasal nerve density with age and no association with gender was confirmed in the present study. In the present study, however, this decline is not as steep as was previously reported. In the study by Niederer et al., ${ }^{28}$ in 85 normal, healthy subjects a $0.9 \%$ annual decline in subbasal nerve density was reported $(r=-0.423, P<0.001)$, while in the present study the annual decline was lower by a factor of three $(0.25-0.30 \%$ ), with a weaker correlation coefficient and significance (manual: $r=-$ $0.211, P=0.04$; auto: $r=-0.247, P=0.01$ ). This difference in result may be due to the relatively few subjects over 60 years of age included in the earlier study, and possible differences in image selection and analysis criteria, which were not explicitly stated in that study. Given the weaker age association and comparatively large standard deviation of nerve density $\left(3.5-6.2 \mathrm{~mm} / \mathrm{mm}^{2}\right)$ reported in the present cohort, caution is warranted in assuming a general age dependence of subbasal nerve density. An association appears to exist for large subject groups, but the result needs confirmation in other large cohorts and cannot be extrapolated to individuals or to small subject groups. In terms of inter-eye correlation of subbasal nerve density, the high correlation we noted may reflect the common denominator of the central nervous system in regulating corneal innervation. As discussed at length in a recent publication, the strong inter-eye correlation extends to bilateral manifestation of corneal nerve pathology in certain cases of unilateral disease. ${ }^{33}$ Our detection of inter-eye correlation of subbasal nerve density in a normal population may therefore have broad consequences for pathology, trauma, or surgical interventions affecting corneal subbasal nerves - namely that subbasal nerve density reduction in one eye may induce a contralateral reduction in subbasal nerves, 
through as yet unidentified mechanisms. More studies investigating the impact of unilateral corneal nerve damage on bilateral subbasal nerve density are required, for example in cases of laser surgery, cross-linking, trauma, or infection.

With a rigorous protocol for nerve tracing, inter-observer agreement in the present study was good, with an observer-dependent density difference of around $2 \%$ on average and no obvious bias detected between trained observers. Agreement of automatically-determined nerve density values with the human observers was somewhat poorer, however, at $9 \%$ on average. Despite these differences, values for subbasal nerve density across observers and between manual and automated methods were consistent for the different age groups (Tables 2 and 3). Moreover, the age-dependence of subbasal nerve density by linear regression analysis yielded similar values and conclusions based on statistical analysis, independent of observer or whether a manual or automatic tracing method was used. This result indicates the potential for automated nerve tracing to replace manual nerve tracing in large patient cohorts, thereby significantly reducing analysis time and avoiding the potential for human bias. Areas for improving the nerve tracing algorithm, however, were identified - specifically in the handling of dendritic cells and short nerve segments with reduced contrast.

A relatively large standard deviation of subbasal nerve density exists at all ages in a normal population, which renders only gross differences in nerve density detectable in clinical studies. Alternatively, for more sensitive detection of pathologic nerve density, large subject groups are required. For example, with a standard deviation of $5 \mathrm{~mm} / \mathrm{mm}^{2}$, to detect a $20 \%$ difference in subbasal nerve density from the normal value would require about 25 patients per group for a statistical power of 0.8 and significance at the $5 \%$ level. Detecting a $10 \%$ difference would require 100 patients per group. This has important implications for clinical studies using subbasal nerve density as a possible prognostic or diagnostic parameter. Early-stage pathology - where IVCM could have the greatest potential for non-invasive screening or diagnosis - could feasibly alter subbasal nerve density at the $10 \%$ level (or less), which would not be detected unless large groups are examined. At the individual patient level, the variability in subbasal nerve density at a given age would make it impossible to use as a single parameter upon which to base a diagnosis. The use of additional subbasal nerve parameters (tortuosity, branching, etc.), qualitative features of nerve architecture, or combinations thereof, however, may be able to provide sensitive and specific indicators on a per-patient basis.

Regarding our study hypothesis, how does the value of subbasal nerve density in the present study compare with previous results obtained with laser-scanning IVCM in healthy populations? In a study with 47 healthy subjects aged $61 \pm 9$ years, Nitoda et al. ${ }^{8}$ reported a subbasal nerve density of $16.6 \pm 4.2 \mathrm{~mm} / \mathrm{mm}^{2}$, which is slightly lower than our 61+ age group (range: $16.7-18.3 \mathrm{~mm} / \mathrm{mm}^{2}$, SD $3.9-5.1 \mathrm{~mm} / \mathrm{mm}^{2}$ ), although different, customized software was used for nerve tracing in that study. In another study by Hertz et al. ${ }^{34}$ with 20 subjects aged $41 \pm 17 \mathrm{y}$, median subbasal nerve density was $16.15 \mathrm{~mm} / \mathrm{mm}^{2}$, determined by custom tracing software and image selection from two fields of view captured in volume scan mode. Conversely, other studies with laser-scanning IVCM have reported a higher value for the mean subbasal nerve density. Niederer et al. ${ }^{35}$ reported a value of $21.6 \pm 6.0 \mathrm{~mm} / \mathrm{mm}^{2}$ in 30 subjects aged $41 \pm 11 \mathrm{y}$, although the image selection criteria were not stated. Another study by Niederer et al. ${ }^{36}$ examined 52 subjects aged $26 \pm 7 y$, and reported a density of $22.4 \pm 6.0 \mathrm{~mm} / \mathrm{mm}^{2}$ based on single observer analysis, with no selection criteria stated (by comparison, density in the youngest age category in this study was $19.4-20.3 \mathrm{~mm} / \mathrm{mm}^{2}$, with SD $3.5-$ $4.2 \mathrm{~mm} / \mathrm{mm}^{2}$ ). Niederer et al., in a third study ${ }^{28}$ with 85 subjects aged $38 \pm 16 y$ (range 18-87y) reported a mean density of $20.3 \pm 6.5 \mathrm{~mm} / \mathrm{mm}^{2}$, but no image selection criteria were stated. Finally, Patel et al. ${ }^{37}$ 
examined 31 subjects aged $35 \pm 12 y$ and reported a density of $25.9 \pm 7.0 \mathrm{~mm} / \mathrm{mm}^{2}$ by selecting a single image per eye with the maximum nerve density. Selecting the maximum nerve density image in the present study (but still excluding the apical whorl region) would have yielded a mean subbasal nerve density of about $23-24 \mathrm{~mm} / \mathrm{mm}^{2}$. Although reported values clearly differ among the various studies, it is believed that a consistent imaging, selection, and analysis protocol would provide better agreement in future studies.

While selecting the single image with the maximum nerve density, if applied consistently, could enable population-based comparisons to be made, the results of this study show that this approach leads to an overestimate of the average central subbasal nerve density by about $15-21 \%$ (and similarly, selecting the single image with the minimum nerve density leads to an underestimate of $15-23 \%$ ). Therefore, to accurately depict the status of the subbasal nerve plexus and to facilitate cross-study comparison, it is recommended to report average density across several images or use a representative image and not the image with the maximum subbasal nerve density. One interesting finding in this study was that choosing a single nerve image randomly per eye for analysis resulted in only a small error (under $5 \%$ in this study) compared to tracing and averaging a mean of 4.3 images/eye. This approach could potentially significantly reduce computation time for the analysis of large data sets, but the result requires confirmation in patient populations with specific pathology. Recently, Vagenas et al. ${ }^{25}$ analyzed subbasal nerves in a group of 20 diabetic patients with nerve pathology, and found that the mean density from 5 randomly-chosen nonoverlapping images of central subbasal nerves sufficed as an estimate of the 'true' mean density (determined from 16 non-overlapping images), with an error of less than $13 \%$ from the true mean $80 \%$ of the time. Regarding an 'optimum' method for image selection, based on this study and others, and a requirement for feasible clinical examination of a large number of patients, an average of density in 3 nonoverlapping, randomly chosen images of the central cornea (meeting the additional image quality criteria outlined in the Methods) is recommended. From Tables 1 and 2, choosing 3 random non-overlapping images from the central cornea would result in a mean error of $1 \%$ (maximum 2\%) compared to 4.3 images/eye.

In terms of imaging mode, it was found in this study that the sequence mode gave greater flexibility, allowing a wider area of the subbasal nerve plexus to be captured in a single scan. Multiple images meeting the outlined selection criteria could obtained from a single sequence scan, whereas a given volume scan typically yielded only 1 useable image of subbasal nerves from a single field of view, out of 40 images taken by the scan. An advantage of the volume scan is that it can enable three-dimensional reconstruction of corneal structures and a finer axial discrimination of morphologic features in the cornea. For the specific goal of subbasal nerve density quantification, however, its limited field of view and relatively long scanning time makes use of the volume scan less practical.

It is worthwhile to recognize that several studies have demonstrated methods for automated wide-field imaging of the subbasal nerve plexus. ${ }^{17-20}$ The resulting montages will undoubtedly aid in subbasal nerve assessment in the future, however, to date these approaches are neither standardized nor commercially available. Moreover, the approaches can be difficult to implement routinely in a clinical setting and may be time- and computation-intensive. It is therefore important to recognize the value of nerve density determination in multiple single frames (with freely available software) as a cost-effective and rapid method to determine an accurate estimate of the true subbasal nerve density in a clinical setting.

What is the clinical relevance of the present study? Although IVCM is a specialized technique available mainly in large academic hospitals and specialized cornea clinics, its use is becoming more widespread. This is in part due to the increasing number of applications of IVCM in assessing clinical conditions, as evidenced 
by the growing scientific literature in this area. Besides the value of ensuring accurate, standardized analysis and reporting of corneal nerve parameters, as well as facilitating the future development of IVCM-based prognostic and diagnostic parameters, the results of this study could be used to gain insight into the clinical variability of parameters influenced by corneal nerves, for example corneal sensitivity, tear film production and quality, and speed of the wound healing response. Given the variability in corneal nerve density in the healthy population, one could expect variability in these clinical parameters in normal subjects. Moreover, the results of the present study lead us to hypothesize that these clinical parameters may be negatively impacted as part of the normal aging process. Further studies could be designed to test such a hypothesis.

It is important to acknowledge limitations of present techniques for nerve density determination. One general limitation of IVCM in determining subbasal nerve density is the variation in the contrast/visibility of subbasal nerves. In some subjects, fine interconnecting branches between nerves were visible, whereas they were invisible in others. This variability could be one source for the large standard deviation of nerve density (and inter-observer discrepancies), and could possibly be mitigated by a more precise definition of nerve structures to include in tracings, based on for example, a contrast threshold or minimum nerve fiber bundle width. In a study of six cadaver corneas analyzed by ex-vivo histochemical staining, ${ }^{3}$ it was shown that these fine interconnecting nerve branches are more numerous than typically visualized by IVCM, with their inclusion resulting in a central corneal subbasal nerve density of $45.94 \pm 5.2 \mathrm{~mm} / \mathrm{mm}^{2}$ when measured ex vivo. Although the IVCM values may not represent the 'true' anatomic subbasal nerve density, the in vivo nature of the measurement is a distinct advantage ${ }^{20}$ and in vivo values have the ability to be compared longitudinally and across centers, provided an appropriate consensus on nerve tracing can be achieved.

A limitation of this study was the deliberate exclusion of the whorl or vortex pattern typically located 2-3mm inferior to the central cornea. ${ }^{3}$ This region was excluded due its known local increase in nerve density ${ }^{17}$ and an imposed requirement to keep the fixation target in a single location during examination (in practice the target must be moved to induce a slight upward gaze in order to visualize the whorl). The whorl region, however, could itself be an important parameter for monitoring and may yield more robust quantitative parameters than the central cornea. Studies specifically examining the whorl region in a large healthy population are warranted, as very little data on subbasal nerve density in this region is available today. ${ }^{17}$

Another limitation of the present study was the potential inclusion of subjects with subclinical alterations not detected by the general ophthalmic examinations. Conditions such as subclinical dry eye, endothelial dystrophy, or inflammation may have influenced the subbasal nerve density. Exclusion of subclinical alterations by additional clinical examination and tests of corneal endothelium, epithelium and tear film could possibly reduce the degree of variation noted in the present study. In addition, a relatively homogeneous Swedish population was examined. The overwhelming majority of subjects were of Caucasian origin, however, ethnicity was not specifically recorded. More information on ethnic and geographic variations in subbasal nerve density is required in order to generalize results. Finally, this was a single-center study with all subjects examined in the same center with the same confocal microscope (Sweden). Manual image selection and tracing were performed at a second site (Norway), and automated analysis at a third site (Italy). Future multi-center studies with image acquisition at multiple sites (using a common protocol) would improve the robustness of the data, and formal establishment of an independent IVCM reading center as recently proposed, ${ }^{38}$ or alternatively, wider adoption of a single automated analysis tool, would be desirable. 
Nevertheless, it is hoped that the current work will serve as a standard for developing further baseline data against which to compare various patient populations with suspected pathologic nerve density. It is also envisioned that a similar framework can be established for the development of additional standardized parameters relating to corneal subbasal nerve morphology.

\section{References}

1. Patel DV, McGhee CNJ. In vivo confocal microscopy of human corneal nerves in health, in ocular and systemic disease, and following corneal surgery: a review. Br J Ophthalmol 2009;93:853-860.

2. Cruzat $A$, Pavan-Langston $D$, Hamrah P. In vivo confocal microscopy of corneal nerves: analysis and clinical correlation. Semin Ophthalmol 2010;25:171-177.

3. Marfurt CF, Cox J, Deek S, Dvorscak L. Anatomy of the human corneal innervation. Exp Eye Res 2010;90:478-492.

4. Guthoff RF, Wienss $H$, Hahnel C, Wree A. Epithelial innervation of human cornea: a three-dimensional study using confocal laser scanning fluorescence microscopy. Cornea 2005;24:608-613.

5. Müller L, Marfurt CF, Kruse F, Tervo TMT. Corneal nerves: structure, contents and function. Exp Eye Res 2003;76:521-542.

6. Pritchard N, Edwards K, Shahidi AM, et al. Corneal markers of diabetic neuropathy. Ocular Surf 2011;9:17-28.

7. Villani E, Galimberti D, Viola F, Mapelli C, Del Papa N, Ratiglia R. Corneal involvement in rheumatoid arthritis: an in vivo confocal study. Invest Ophthalmol Vis Sci 2008;49:560-4.

8. Nitoda $\mathrm{E}$, Kallinikos $\mathrm{P}$, Pallikaris $\mathrm{A}$, et al. Correlation of diabetic retinopathy and corneal neuropathy using confocal microscopy. Curr Eye Res 2012;37:898-906.

9. Deng SX, Sejpal KD, Tang Q, Aldave AJ, Lee OL, Yu F. Characterization of limbal stem cell deficiency by in vivo laser scanning confocal microscopy. Arch Ophthalmol 2012;130:440-445.

10. Miri A, Alomar T, Nubile M, Al-aqaba M, et al. In vivo confocal microscopic findings in patients with limbal stem cell deficiency. Br J Ophthalmol 2012;96:523-529.

11. Eden U, Fagerholm P, Danyali R, Lagali N. Pathologic epithelial and anterior corneal nerve morphology in early-stage congenital aniridic keratopathy. Ophthalmology 2012;119:1803-10.

12. Zhang M, Chen J, Luo L, Xiao $Q$, Sun M, Liu Z. Altered corneal nerves in aqueous tear deficiency viewed by in vivo confocal microscopy. Cornea 2005;24:818-24.

13. Benítez-Del-Castillo JM, Acosta MC, Wassfi MA, Díaz-Valle D, Gegúndez JA, Fernandez C, García-Sánchez J. Relation between corneal innervation with confocal microscopy and corneal sensitivity with noncontact esthesiometry in patients with dry eye. Invest Ophthalmol Vis Sci 2007;48:173-81.

14. Hamrah P, Cruzat A, Dastjerdi MH, Prüss H, Zheng L, Shahatit BM, Bayhan HA, Dana R, Pavan-Langston D. Unilateral herpes zoster ophthalmicus results in bilateral corneal nerve alteration: an in vivo confocal microscopy study. Ophthalmology 2013;120:40-7.

15. Ahuja Y, Baratz KH, McLaren JW, Bourne WM, Patel SV. Decreased corneal sensitivity and abnormal corneal nerves in Fuchs endothelial dystrophy. Cornea 2012;31:1257-63.

16. Schrems-HoesI LM, Schrems WA, Cruzat A, Shahatit BM, Bayhan HA, Jurkunas UV, Hamrah P. Cellular and subbasal nerve alterations in early stage Fuchs' endothelial corneal dystrophy: an in vivo confocal microscopy study. Eye 2013;27(1):42-9. 
17. Patel DV, McGhee CNJ. Mapping of the normal human corneal sub-basal nerve plexus by in vivo laser scanning confocal microscopy. Invest Ophthalmol Vis Sci 2005;46:4485-4488.

18. Zhivov A, Blum M, Guthoff $R$, Stachs $O$. Real-time mapping of the subepithelial nerve plexus by in vivo confocal laser scanning microscopy. Br J Ophthalmol 2010;94:1133-1135.

19. Edwards K, Pritchard N, Gosschalk K, Sampson GP, Russell A, Malik RA, Efron N. Wide-field assessment of the human corneal subbasal nerve plexus in diabetic neuropathy using a novel mapping technique. Cornea 2012;31:1078-1082.

20. Turuwhenua JT, Patel DV, McGhee CNJ. Fully automated montaging of laser scanning in vivo confocal microscopy images of the human corneal subbasal nerve plexus. Invest Ophthalmol Vis Sci 2012;53:2235-2242.

21. Allgeier S, Zhivov A, Eberle F, Koehler B, Maier S, Bretthauer G, Guthoff RF, Stachs O. Image reconstruction of the subbasal nerve plexus with in vivo confocal microscopy. Invest Ophthalmol Vis Sci 2011;52:5022-8.

22. Scarpa F, Grisan E, Ruggeri A. Automatic recognition of corneal nerve structures in images from confocal microscopy. Invest Ophthalmol Vis Sci 2008;49:4801- 4807.

23. Guthoff RF, Zhivov A, Stachs O. In vivo confocal microscopy, an inner vision of the cornea - a major review. Clin Experiment Ophthalmol 2009;37:100-117.

24. Niederer RL, McGhee CN. Clinical in vivo confocal microscopy of the human cornea in health and disease. Prog Retin Eye Res 2010;29:30-58.

25. Vagenas D, Pritchard N, Edwards K, Shahidi AM, Sampson GP, Russell AW, Malik RA, Efron N. Optimal image sample size for corneal nerve morphometry. Optom Vis Sci 2012;89:812-817.

26. Scarpa $F$, Zheng $X$, Ohashi $Y$, Ruggeri A. Automatic evaluation of corneal nerve tortuosity in images from in vivo confocal microscopy. Invest Ophthalmol Vis Sci 2011;52:6404-8.

27. Kallinikos P, Berhanu M, O'Donnell C, Boulton AJM, Efron N, Malik RA. Corneal nerve tortuosity in diabetic patients with neuropathy. Invest Ophthalmol Vis Sci 2004;45:418-422.

28. Niederer RL, Perumal D, Sherwin T, McGhee CNJ. Age-related differences in the normal human cornea: a laser scanning in vivo confocal microscopy study. Br J Ophthalmol 2007;91:1165-1169.

29. Patel DV, McGhee CNJ. In vivo laser scanning confocal microscopy confirms that the human corneal subbasal nerve plexus is a highly dynamic structure. Invest Ophthalmol Vis Sci 2008;49:3409-3412.

30. Meijering E, Jacob M, Sarria JCF, Steiner $P$, Hirling $H$, Unser M. Design and validation of a tool for neurite tracing and analysis in fluorescence microscopy images. Cytometry Pt A 2004;58:167-176.

31. Lagali NS, Griffith M, Shinozaki N, Fagerholm P, Munger R. Innervation of tissue-engineered corneal implants in a porcine model: a 1-year in vivo confocal microscopy study. Invest Ophthalmol Vis Sci 2007;48:3537-44.

32. Bland JM, Altman DG. Statistical methods for assessing agreement between two methods of clinical measurement. Lancet 1986;i:307-10.

33. Yamaguchi T, Turhan A, Harris DL, Hu K, Prüss H, von Andrian U, Hamrah P. Bilateral nerve alterations in a unilateral experimental neurotrophic keratopathy model: a lateral conjunctival approach for trigeminal axotomy. PLoS One. 2013;8(8):e70908

34. Hertz P, Bril V, Orszag A, Ahmed A, Ng E, Nwe P, Ngo M, Perkins BA. Reproducibility of in vivo corneal confocal microscopy as a novel screening test for early diabetic sensorimotor polyneuropathy. Diabet Med 2011;28:1253-1260.

35. Niederer RL, Perumal D, Sherwin T, McGhee CNJ. Corneal innervation and cellular changes after corneal transplantation: an in vivo confocal microscopy study. Invest Ophthalmol Vis Sci 2007;48:621-626. 
36. Niederer RL, Perumal D, Sherwin T, McGhee CN. Laser scanning in vivo confocal microscopy reveals reduced innervation and reduction in cell density in all layers of the keratoconic cornea. Invest Ophthalmol Vis Sci 2008;49:2964-70.

37. Patel DV, Ku JY, Johnson R, McGhee CN. Laser scanning in vivo confocal microscopy and quantitative aesthesiometry reveal decreased corneal innervation and sensation in keratoconus. Eye 2009;23:586-92.

38. Patel DV, McGhee CN. Quantitative analysis of in vivo confocal microscopy images: a review. Surv Ophthalmol 2013 (publish ahead of print). 
Table 1. Demographic characteristics for the population of healthy subjects examined.

Total group

\begin{tabular}{|c|c|c|c|c|}
\hline $\begin{array}{l}\text { No. of subjects } \\
\text { female } \\
\text { male }\end{array}$ & \multicolumn{4}{|c|}{$\begin{array}{c}106 \\
59 \\
47\end{array}$} \\
\hline $\begin{array}{l}\text { Mean age, y } \\
\text { (range) } \\
\text { female } \\
\text { male }\end{array}$ & & $\begin{array}{l}50.01 \\
50.61 \\
48.7(\end{array}$ & $\begin{array}{l}88) \\
82) \\
88)\end{array}$ & \\
\hline Age group & $15-30$ & $31-45$ & $46-60$ & $61+$ \\
\hline $\begin{array}{l}\text { no. of subjects } \\
\text { female } \\
\text { male } \\
\text { mean age }(y)\end{array}$ & $\begin{array}{c}24 \\
12 \\
12 \\
25.2\end{array}$ & $\begin{array}{c}24 \\
10 \\
14 \\
37.3\end{array}$ & $\begin{array}{c}21 \\
17 \\
4 \\
52.4\end{array}$ & $\begin{array}{c}37 \\
20 \\
17 \\
72.4\end{array}$ \\
\hline
\end{tabular}


Table 2. Influence of nerve calculation method, observer, and age group on mean and standard deviation of nerve density.

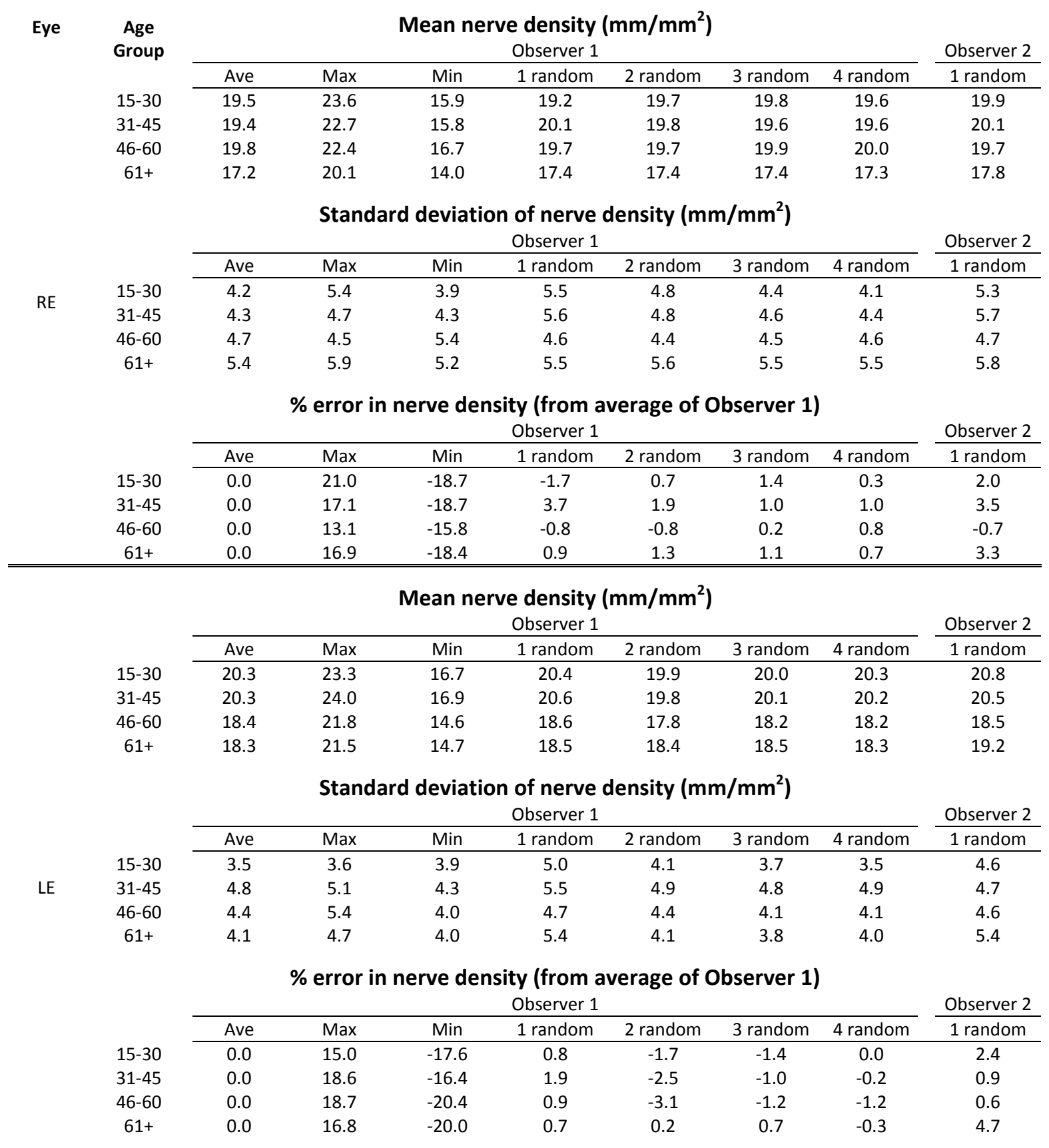

$\mathrm{RE}=$ right eye; $\mathrm{LE}=$ left eye. Density calculation method for each eye: Ave = mean of density in all selected images; $M a x=$ single image with maximum nerve density; $M i n=$ single image with minimum nerve density; 1-4 random = mean nerve density across 1-4 randomly selected images. 
Table 3. Influence of nerve calculation method and age group on mean and standard deviation of nerve density determined by the automated nerve tracing method. $\mathrm{RE}=$ right eye; $\mathrm{LE}=$ left eye.

\begin{tabular}{|c|c|c|c|c|c|c|c|c|}
\hline \multirow[t]{8}{*}{ Eye } & \multirow[t]{2}{*}{$\begin{array}{l}\text { Age } \\
\text { Group }\end{array}$} & \multicolumn{7}{|c|}{ Mean nerve density $\left(\mathrm{mm} / \mathrm{mm}^{2}\right)$} \\
\hline & & Ave & Max & Min & 1 random & 2 random & 3 random & 4 random \\
\hline & $15-30$ & 19.4 & 23.4 & 15.6 & 20.3 & 19.7 & 19.6 & 19.4 \\
\hline & $31-45$ & 19.6 & 22.7 & 16.5 & 20.5 & 20.1 & 19.8 & 19.8 \\
\hline & $46-60$ & 20.1 & 22.9 & 16.8 & 19.9 & 20.0 & 20.2 & 20.3 \\
\hline & $61+$ & 16.7 & 20.2 & 13.4 & 17.4 & 17.1 & 16.8 & 16.8 \\
\hline & & \multicolumn{7}{|c|}{$\begin{array}{l}\text { Standard deviation of nerve density }\left(\mathrm{mm} / \mathrm{mm}^{2}\right) \\
\text { Automated }\end{array}$} \\
\hline & & Ave & Max & Min & 1 random & 2 random & 3 random & 4 random \\
\hline \multirow{11}{*}{$\mathrm{RE}$} & $15-30$ & 4.0 & 5.1 & 4.0 & 5.0 & 4.5 & 4.0 & 3.8 \\
\hline & $31-45$ & 4.2 & 4.7 & 4.5 & 5.5 & 4.3 & 4.5 & 4.3 \\
\hline & $46-60$ & 4.5 & 4.7 & 4.9 & 4.9 & 4.0 & 4.3 & 4.4 \\
\hline & $61+$ & 5.5 & 6.2 & 5.6 & 6.0 & 5.6 & 5.6 & 5.5 \\
\hline & & \multicolumn{7}{|c|}{$\%$ error in nerve density (from average of automated) } \\
\hline & & \multicolumn{7}{|c|}{ Automated } \\
\hline & & Ave & Max & Min & 1 random & 2 random & 3 random & 4 random \\
\hline & $15-30$ & 0.0 & 20.7 & -19.6 & 4.7 & 1.5 & 0.9 & -0.1 \\
\hline & $31-45$ & 0.0 & 15.6 & -15.8 & 4.5 & 2.7 & 1.0 & 1.2 \\
\hline & $46-60$ & 0.0 & 14.2 & -16.2 & -0.8 & -0.5 & 0.9 & 1.1 \\
\hline & $61+$ & 0.0 & 20.8 & -19.7 & 4.3 & 2.7 & 0.6 & 0.7 \\
\hline \multirow{20}{*}{ LE } & & \multicolumn{7}{|c|}{ Mean nerve density $\left(\mathrm{mm} / \mathrm{mm}^{2}\right)$} \\
\hline & & Ave & Max & Min & 1 random & 2 random & 3 random & 4 random \\
\hline & $15-30$ & 20.2 & 23.4 & 16.9 & 20.6 & 19.8 & 19.8 & 20.2 \\
\hline & $31-45$ & 20.2 & 23.5 & 16.8 & 20.3 & 19.7 & 20.1 & 20.2 \\
\hline & $46-60$ & 18.0 & 21.9 & 14.0 & 17.8 & 17.2 & 17.8 & 17.9 \\
\hline & $61+$ & 18.2 & 21.9 & 14.0 & 18.1 & 18.4 & 18.3 & 18.2 \\
\hline & & \multicolumn{7}{|c|}{ Standard deviation of nerve density $\left(\mathrm{mm} / \mathrm{mm}^{2}\right)$} \\
\hline & & & & & Automate & & & \\
\hline & & Ave & Max & Min & 1 random & 2 random & 3 random & 4 random \\
\hline & $15-30$ & 3.5 & 3.5 & 4.1 & 4.5 & 3.9 & 3.9 & 3.5 \\
\hline & $31-45$ & 4.5 & 5.2 & 4.3 & 4.8 & 4.6 & 4.4 & 4.6 \\
\hline & $46-60$ & 4.2 & 5.5 & 4.2 & 4.4 & 4.4 & 4.1 & 4.1 \\
\hline & $61+$ & 3.9 & 4.5 & 4.4 & 5.4 & 4.2 & 3.9 & 3.9 \\
\hline & & \multirow{2}{*}{\multicolumn{7}{|c|}{$\begin{array}{c}\% \text { error in nerve density (from average of automated) } \\
\text { Automated }\end{array}$}} \\
\hline & & & & & & & & \\
\hline & & Ave & Max & Min & 1 random & 2 random & 3 random & 4 random \\
\hline & $15-30$ & 0.0 & 15.6 & -16.5 & 1.6 & -1.9 & -2.0 & -0.1 \\
\hline & $31-45$ & 0.0 & 16.6 & -16.6 & 0.7 & -2.3 & -0.3 & 0.0 \\
\hline & $46-60$ & 0.0 & 21.2 & -22.4 & -1.2 & -4.6 & -1.6 & -1.1 \\
\hline & $61+$ & 0.0 & 20.0 & -23.3 & -0.4 & 1.0 & 0.6 & -0.3 \\
\hline
\end{tabular}

$\mathrm{RE}=$ right eye; $\mathrm{LE}=$ left eye. Density calculation method for each eye: Ave = mean of density in all selected images; $M a x=$ single image with maximum nerve density; $M i n=$ single image with minimum nerve density; 1-4 random = mean nerve density across 1-4 randomly selected images. 
Table 4. Results of linear regression of nerve density vs. age, for different eyes, observers, density calculation methods, and tracing methods (manual and automated).

\begin{tabular}{|c|c|c|c|c|c|c|c|c|c|}
\hline Eye & Observer & $\begin{array}{c}\text { Calculation } \\
\text { Method } \\
\end{array}$ & $\begin{array}{c}\text { Slope } \\
\text { (\% loss/year) } \\
\end{array}$ & $\begin{array}{l}y \text {-intercept } \\
\left(\mathrm{mm} / \mathrm{mm}^{2}\right) \\
\end{array}$ & $R^{2}$ & Observer & $\begin{array}{c}\text { Slope } \\
\text { (\% loss/year) } \\
\end{array}$ & $\begin{array}{l}y \text {-intercept } \\
\left(\mathrm{mm} / \mathrm{mm}^{2}\right) \\
\end{array}$ & $\mathrm{R}^{2}$ \\
\hline \multirow{9}{*}{ RE } & \multirow{8}{*}{1} & All & 0.25 & 21.4 & 0.03 & \multirow{8}{*}{ Auto } & 0.30 & 21.1 & 0.03 \\
\hline & & Average & 0.25 & 21.2 & 0.04 & & 0.31 & 21.7 & 0.06 \\
\hline & & Max & 0.37 & 25.6 & 0.07 & & 0.34 & 25.4 & 0.06 \\
\hline & & Min & 0.19 & 17.3 & 0.02 & & 0.28 & 18.1 & 0.05 \\
\hline & & 1 random & 0.23 & 21.2 & 0.03 & & 0.33 & 22.5 & 0.05 \\
\hline & & 2 random & 0.25 & 21.4 & 0.04 & & 0.30 & 21.9 & 0.06 \\
\hline & & 3 random & 0.26 & 21.5 & 0.04 & & 0.31 & 21.9 & 0.06 \\
\hline & & 4 random & 0.25 & 21.4 & 0.04 & & 0.30 & 21.8 & 0.06 \\
\hline & 2 & 1 random & 0.24 & 21.6 & 0.03 & & & & \\
\hline \multirow{9}{*}{ LE } & \multirow{8}{*}{1} & All & 0.27 & 22.1 & 0.04 & \multirow{8}{*}{ Auto } & 0.35 & 22.4 & 0.05 \\
\hline & & Average & 0.25 & 21.7 & 0.05 & & 0.27 & 21.7 & 0.06 \\
\hline & & Max & 0.24 & 25.0 & 0.04 & & 0.21 & 24.7 & 0.03 \\
\hline & & Min & 0.28 & 18.4 & 0.07 & & 0.36 & 18.9 & 0.10 \\
\hline & & 1 random & 0.24 & 21.7 & 0.03 & & 0.28 & 21.8 & 0.05 \\
\hline & & 2 random & 0.20 & 20.9 & 0.03 & & 0.20 & 20.7 & 0.03 \\
\hline & & 3 random & 0.20 & 21.1 & 0.04 & & 0.22 & 21.2 & 0.04 \\
\hline & & 4 random & 0.25 & 21.6 & 0.05 & & 0.27 & 21.7 & 0.07 \\
\hline & 2 & 1 random & 0.21 & 21.8 & 0.03 & & & & \\
\hline
\end{tabular}

$R E=$ right eye; $L E=$ left eye. $R^{2}$ = quality of fit of the regression line (square of linear correlation coefficient $r$ ). Regression calculation method for each eye: All = use density values in all images selected for analysis (no averaging); Ave = use single data point per eye (mean of density in all selected images for that eye); Max = single data point per eye from image with maximum nerve density; Min = single data point per eye from image with minimum nerve density; 1-4 random = single data point per eye from mean nerve density across 1-4 randomly selected images. 

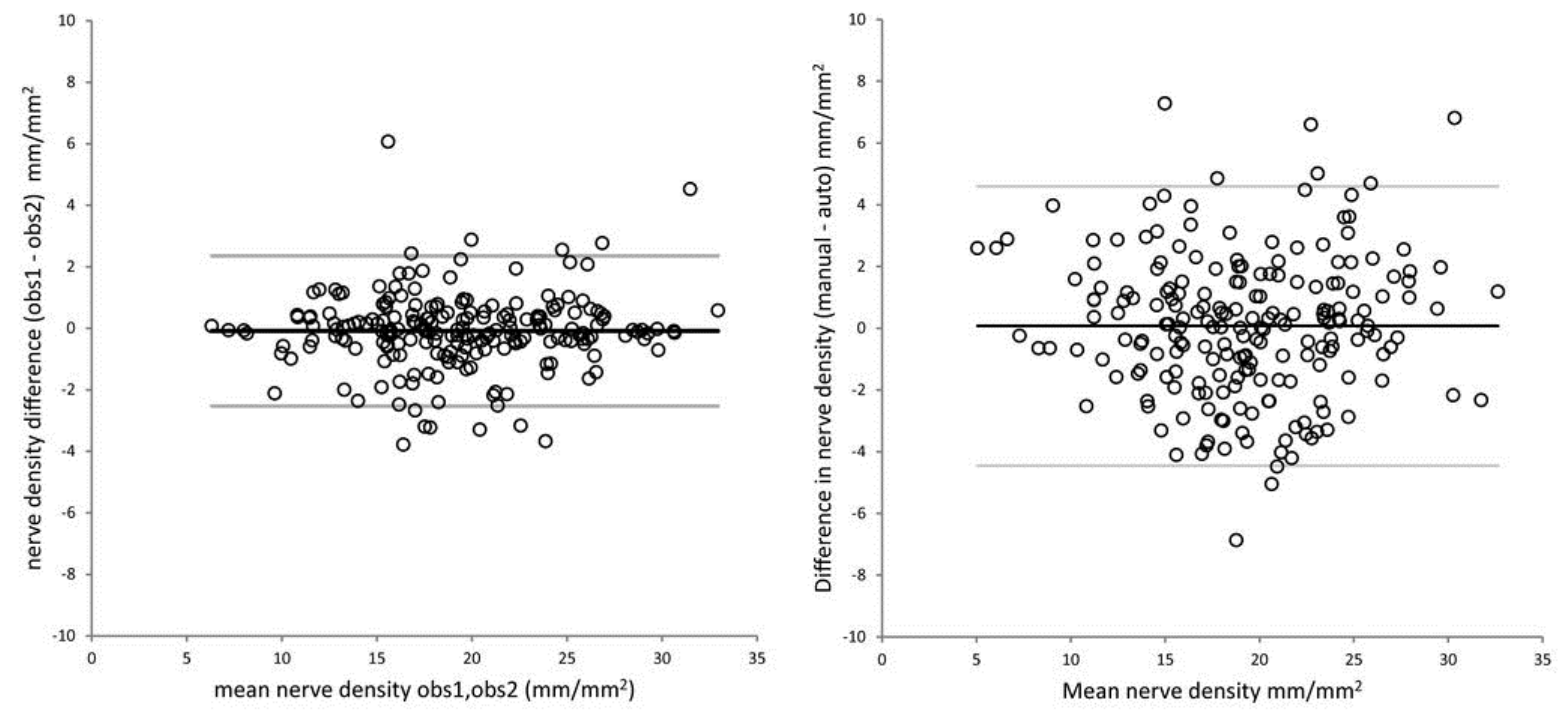

Figure 1. Analysis of inter-observer and inter-method agreement by the Bland-Altman technique. Agreement between human observers (left plot) was good, indicated by the clustering of differences around the value of 0 , and narrow $95 \%$ LOA $\left( \pm 2.44 \mathrm{~mm} / \mathrm{mm}^{2}\right.$, grey lines) around the mean difference (black line). Agreement between manual (observer 1) and automated methods of nerve density assessment (right plot) was somewhat poorer, with a wider distribution of differences around 0, and a 95\% LOA $\left( \pm 4.52 \mathrm{~mm} / \mathrm{mm}^{2}\right)$ almost twice as wide as the manual result. 

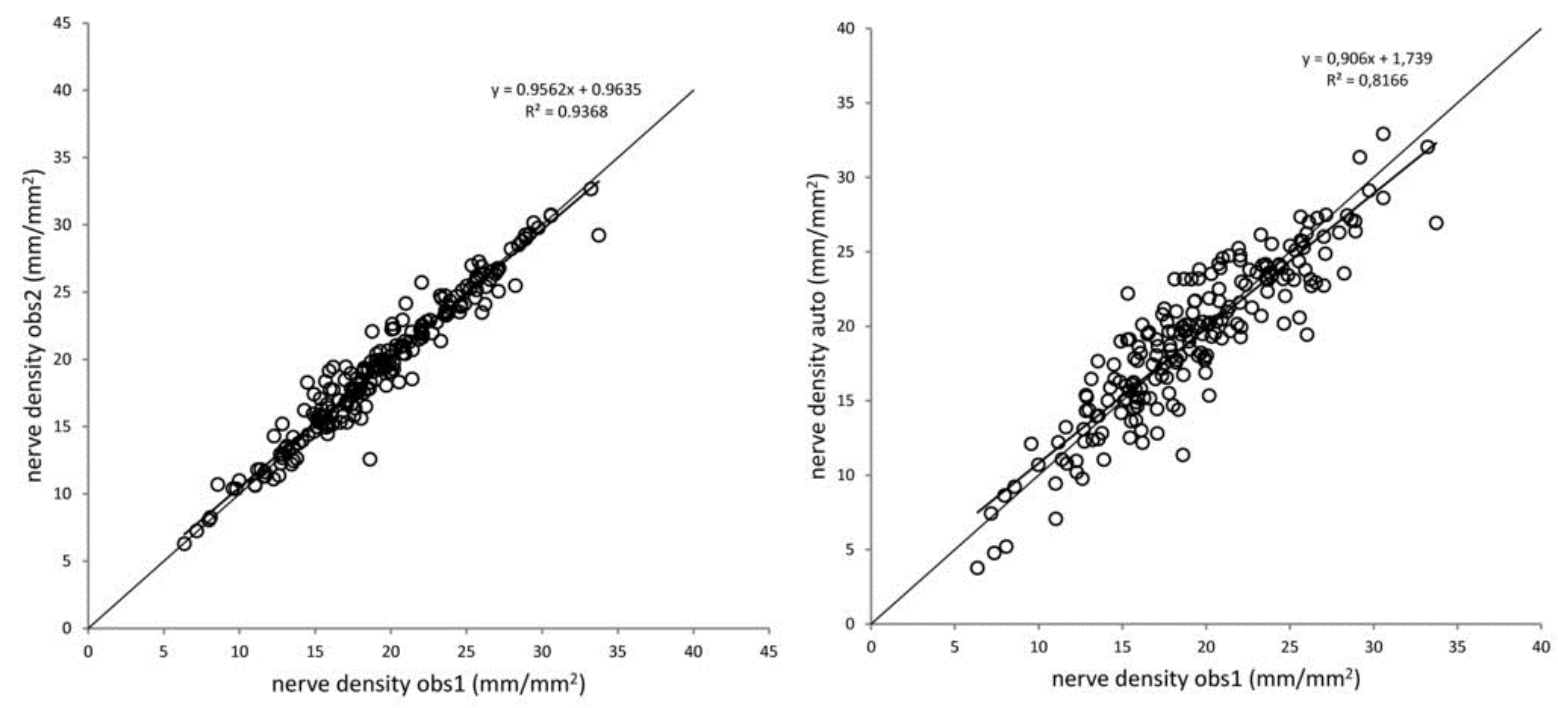

Figure 2. Linearity and correlation of inter-observer (left plot) and inter-method (right plot) measures of nerve density. Good linearity and a high correlation of density values were evident in both cases. 
Original

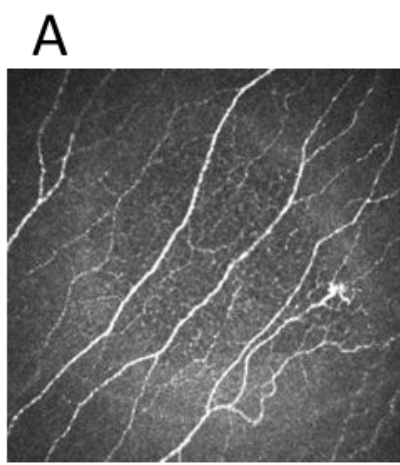

B

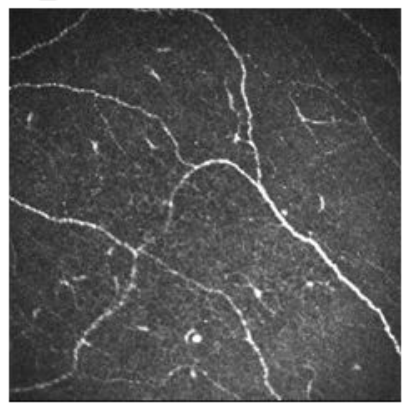

C

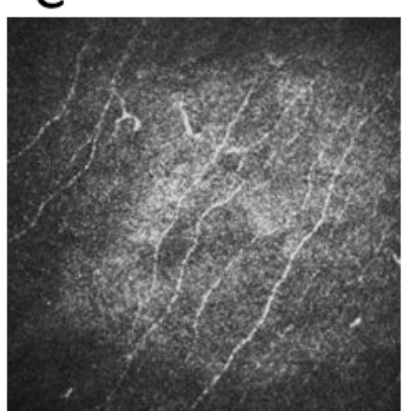

Observer 1

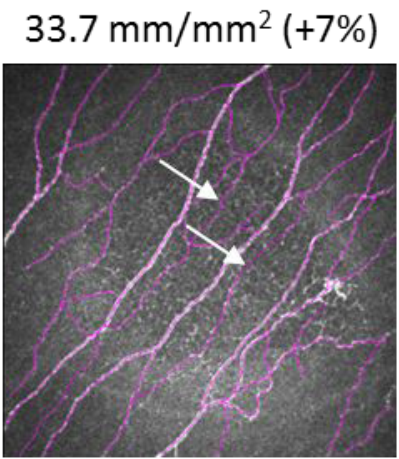

$14.5 \mathrm{~mm} / \mathrm{mm}^{2}(-12 \%)$

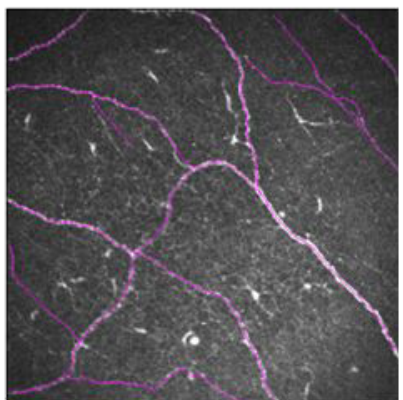

$18.6 \mathrm{~mm} / \mathrm{mm}^{2}(+19 \%)$
Observer 2

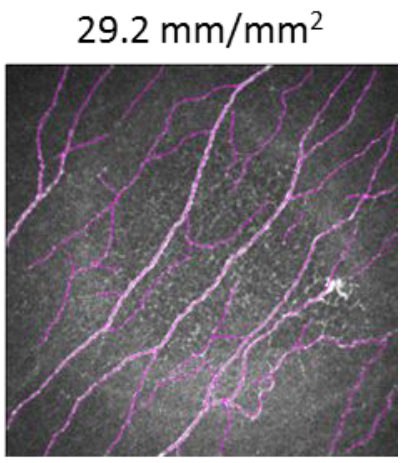

$18.2 \mathrm{~mm} / \mathrm{mm}^{2}$

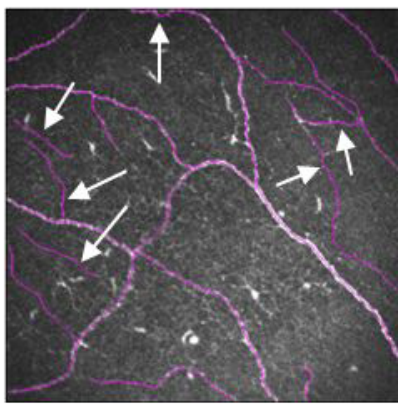

$12.6 \mathrm{~mm} / \mathrm{mm}^{2}$
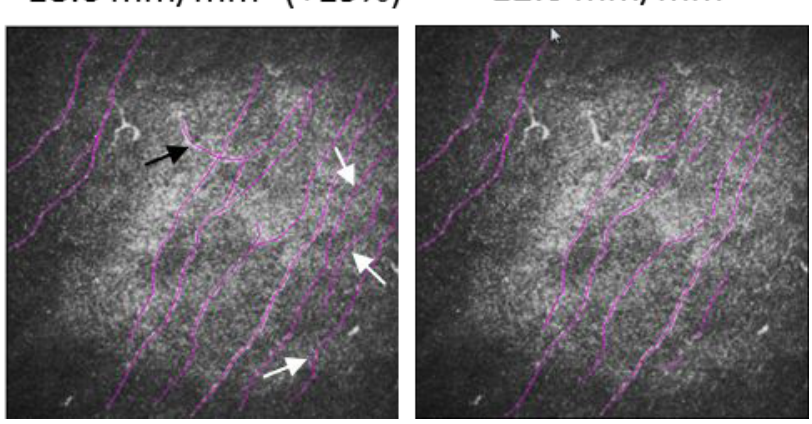

Figure 3. Three images with large nerve density differences between two human observers. The original images are given along with the traced images for both observers. Nerve density values given correspond to the tracings and determination of total nerve length in NeuronJ. Values in parentheses indicate the percentage difference in nerve density between observers, from the mean value. (A) Nerve fibers with reduced contrast (arrows) are included by Observer 1 but not Observer 2. (B) Short nerve branches with reduced contrast (arrows) are included by Observer 2 but not Observer 1. (C) A dendritic cell (black arrow) and several reduced-contrast nerve fibers (white arrows) are included by Observer 1 but not Observer 2 . Note the increased background reflectivity in the image. 


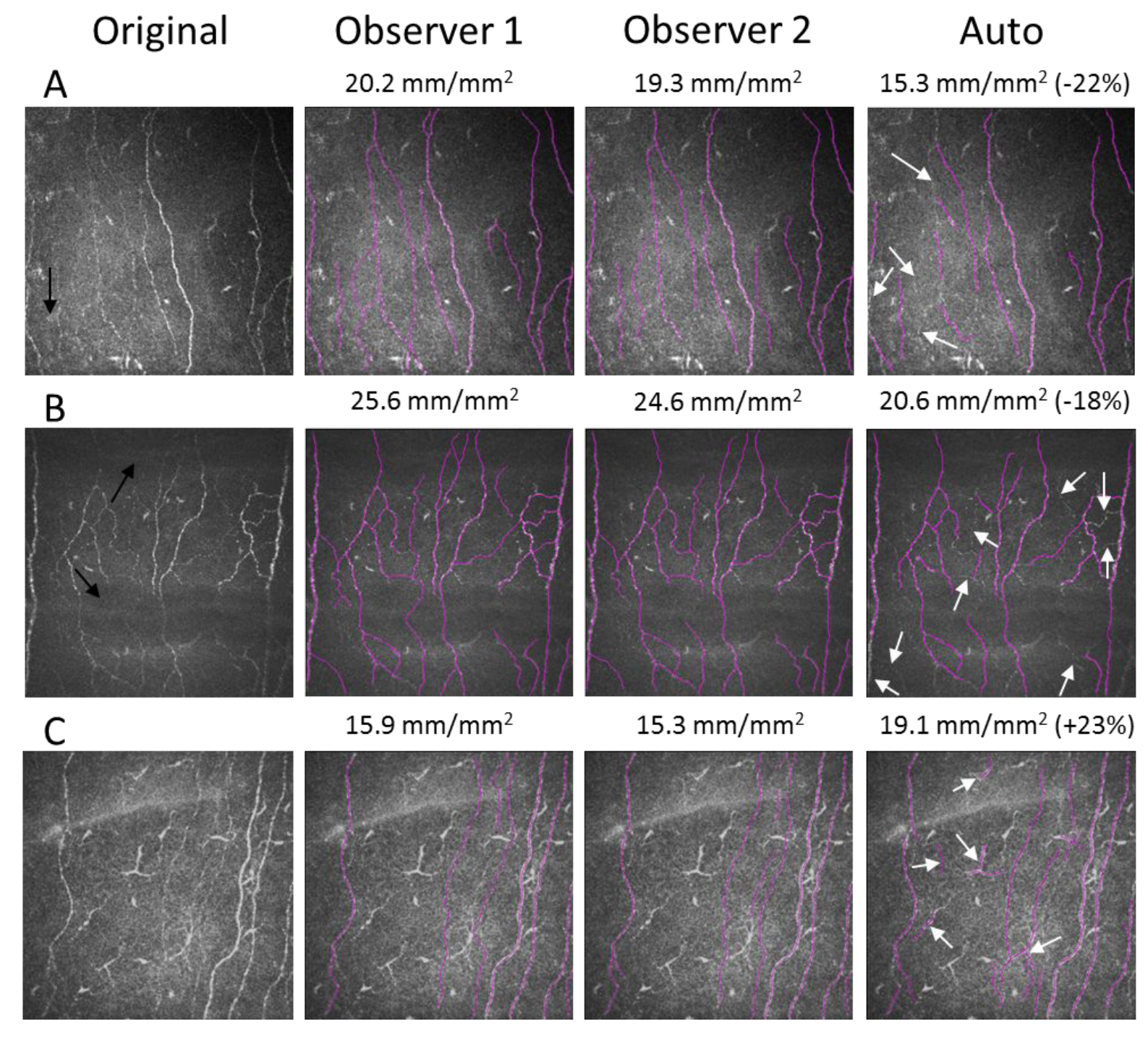

Figure 4. Three images with large differences in nerve density between automatic and manual methods of nerve tracing, but with small inter-observer differences. (A) Slightly oblique image, with anterior keratocytes (black arrow) visible in the same plane as nerves. Automatic nerve tracing excluded several nerve segments with reduced contrast (white arrows) that were included by both human observers. (B) Image with pressure artifacts (black arrows). Despite these artifacts, nerve segments crossing the artifacts were detected and included by both human observers and the automated method. A number of short, reduced-contrast nerve segments (white arrows) were excluded by the automated method but included by human observers. (C) Image with dendritic cells bearing dendrites. While both human observers excluded dendritic cells, many dendrites were included as nerves (white arrows) in the automated method, leading to an overestimate of nerve density. 

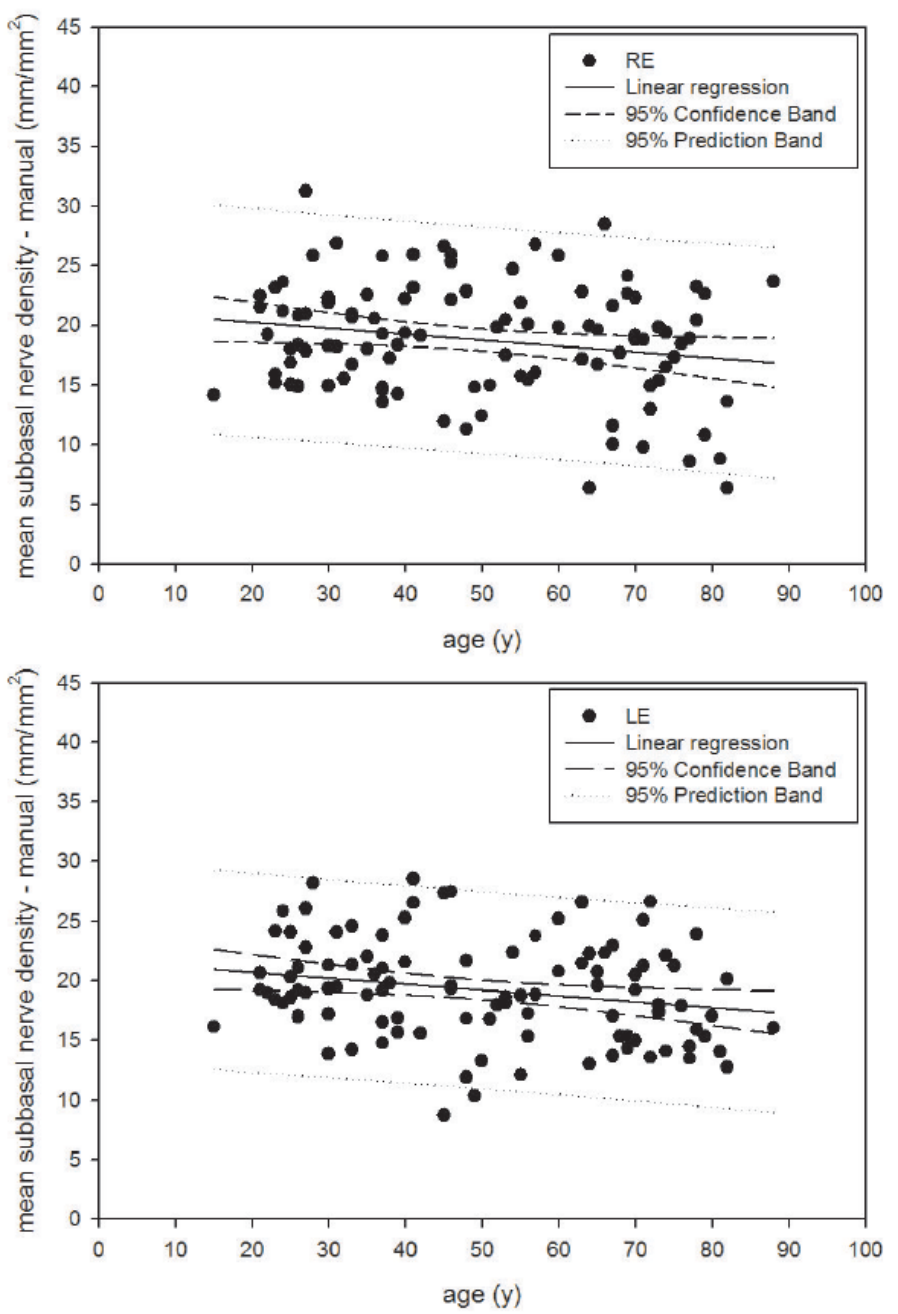

Figure 5. Variation of subbasal nerve density with age in the right eye (RE, upper plot) and left eye (LE, lower plot) as determined by manual nerve tracing. Data is the mean of two independent observers. The linear regression line and $95 \%$ confidence and prediction bands are indicated. A significant negative correlation of subbasal nerve density with age was found independent of eye. The standard deviation of nerve density did not vary substantially with age. 

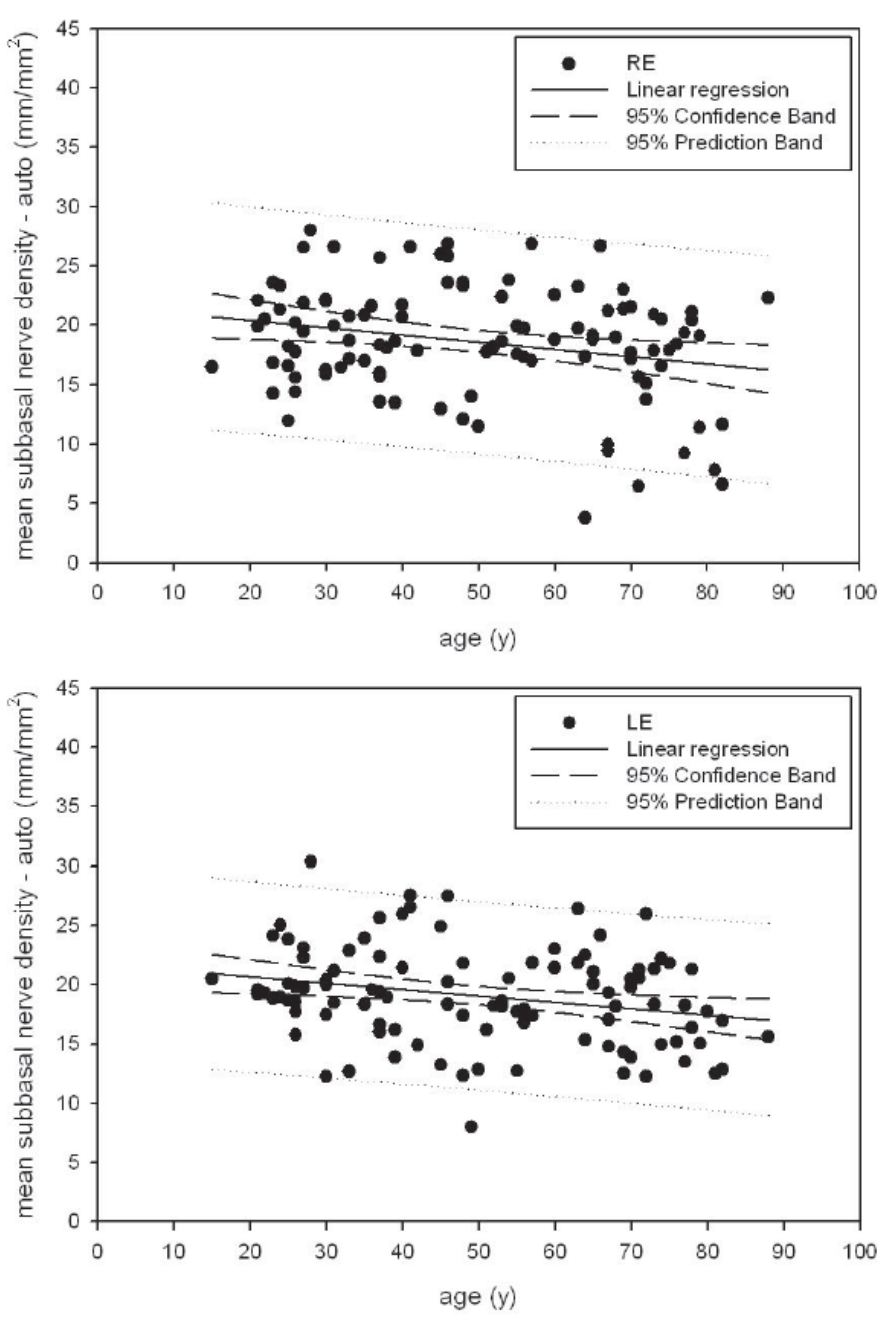

Figure 6. Variation of subbasal nerve density with age in the right eye (RE, upper plot) and left eye (LE, lower plot) as determined by automated nerve tracing. The linear regression line and $95 \%$ confidence and prediction bands are indicated. A significant negative correlation of subbasal nerve density with age was found independent of eye. The standard deviation of nerve density did not vary substantially with age. 

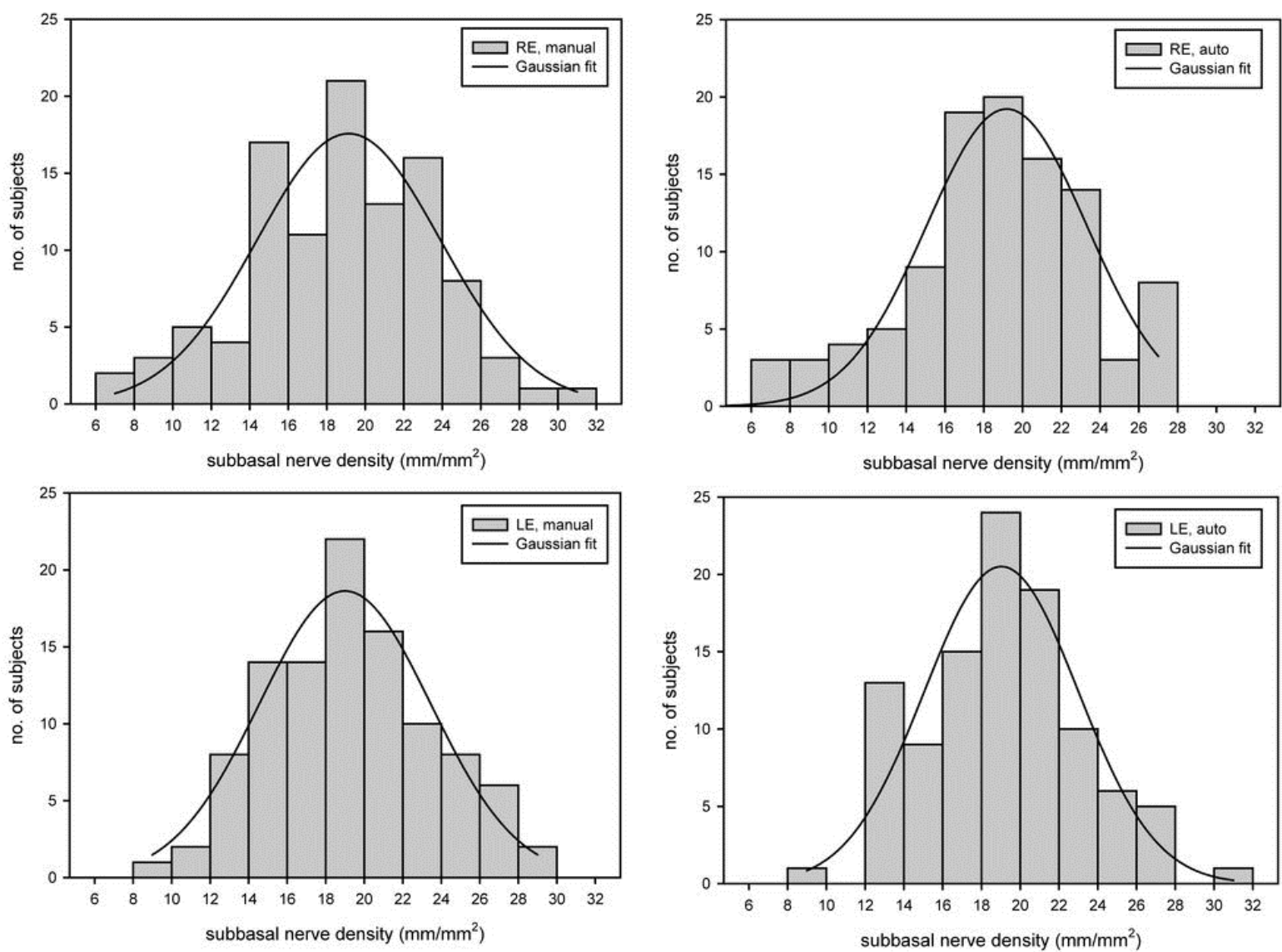

Figure 7. Distribution of subbasal nerve density among 106 healthy subjects, determined by manual (left) and automatic (right) nerve tracing for right eyes (RE, top) and left eyes (LE, bottom). The best-fit Gaussian curve by nonlinear regression is indicated. The most frequent value of subbasal nerve density ranged from $19.0-19.2 \mathrm{~mm} / \mathrm{mm}^{2}$, independent of eye or tracing method. 